\title{
Slc7a11 Modulated by POU2F1 is Involved in Pigmentation in Rabbit
}

\author{
Yang Chen ${ }^{1,2}$, Shuaishuai Hu ${ }^{1}$, Lin Mu ${ }^{1}$, Bohao Zhao ${ }^{1}$, Manman Wang ${ }^{1}$, Naisu Yang ${ }^{1}$, \\ Guolian Bao ${ }^{3}$, Cigen $\mathrm{Zhu}^{4}$ and Xinsheng $\mathrm{Wu}{ }^{1,2, *,+}$ (D) \\ 1 College of Animal Science and Technology, Yangzhou University, Yangzhou 225009, China; \\ yangc@yzu.edu.cn (Y.C.); 18852726848@163.com (S.H.); mulin912@126.com (L.M.); \\ zhao598841633@163.com (B.Z.); wmm171717@126.com (M.W.); yangnaisu@foxmail.com (N.Y.) \\ 2 Joint International Research Laboratory of Agriculture \& Agri-Product Safety, Yangzhou University, \\ Yangzhou 225009, China \\ 3 Animal Husbandry and Veterinary Research Institute, Zhejiang Academy of Agricultural Sciences, \\ Hangzhou 310000, China; GuolianB@126.com \\ 4 Jinling Rabbit Farm, Nanjing 210005, China; js_zhucigen@foxmail.com \\ * Correspondence: xswu@yzu.edu.cn \\ + Current affiliation: College of Animal Science and Technology, Yangzhou University, 48 South University \\ Ave Yangzhou, Yangzhou 225009, China.
}

Received: 24 April 2019; Accepted: 18 May 2019; Published: 20 May 2019

check for updates

\begin{abstract}
Solute carrier family 7 member 11 (Slc7a11) is a cystine/glutamate xCT transporter that controls the production of pheomelanin pigment to change fur and skin color in animals. Previous studies have found that skin expression levels of Slc7a11 varied significantly with fur color in Rex rabbits. However, the molecular regulation mechanism of Slc7a11 in pigmentation is unknown. Here, rabbit melanocytes were first isolated and identified. The distribution and expression pattern of Slc7a11 was confirmed in skin from rabbits with different fur colors. Slc7a11 affected the expression of pigmentation related genes and thus affected melanogenesis. Meanwhile, Slc7a11 decreased melanocyte apoptosis, but inhibition of Slc7a11 enhanced apoptosis. Furthermore, the POU2F1 protein was found to bind to the -713 to -703 bp region of Slc7a11 promoter to inhibit its activity in a dual-luciferase reporter and site-directed mutagenesis assay. This study reveals the function of the Slc7a11 in melanogenesis and provides in-depth analysis of the mechanism of fur pigmentation.
\end{abstract}

Keywords: Rex Rabbit; melanocyte; pigmentation; Slc7a11; POU2F1

\section{Introduction}

The fur color of mammals mainly depends on melanin deposition, and melanogenesis is mainly regulated by melanocytes. The production of different types of melanin by melanocytes, together with different distribution of these pigments, result in a variety of hair colors in mammals [1]. Related genes, such as TYR, TYRP1, ASIP, MITF, and CREB1, have been found to regulate melanin deposition [2,3]. Previously, by using the transcriptome sequencing (RNA-Seq), a significant difference was found in the expression of the Slc7a11 gene in the skin of rabbits with different fur colors. It was speculated to be involved in fur pigmentation [4].

In the melanogenesis pathway, both eumelanin and pheomelanin are derived from a common precursor named dopaquinone [5]. Cystine or glutathione is required for the production of pheomelanin and it is $\mathrm{xCT}$, the protein encoded by the Slc7a11 gene, that acts as a vector to transport extracellular cystine into the cell and maintain normal intracellular glutathione levels. Pheomelanin and eumelanin together form a mixed pigment that determines the skin and fur color of animals [6-8]. In the hair of Slc7a11 gene-mutated mice (sut), the level of pheomelanin was significantly decreased, while the 
eumelanin level was substantially unchanged, so that the wild-type mice with yellow background appeared gray [9]. The sut mutation results in a huge deletion in the Slc7a11 gene, but similar deletions could not be found in this region of Rex Rabbits with six different fur colors, including black (BL), chinchilla $(\mathrm{CH})$, white $(\mathrm{WH})$, brown (BR), protein yellow (PY), and protein chinchilla (PC). SNPs in the exon region of Slc7a11 were also scanned, but no mutation site was found. This indicates that Slc7a11 is highly conserved in the population (data not shown). Currently, studies on the functions of Slc7a11 mainly focus on its important roles in cell proliferation [10], oxidative stress response [11], and Alzheimer's disease target treatment [12]. Research studying its regulatory mechanisms is focused on microRNAs affecting cancer development and apoptosis by targeting and regulating Slc7a11 [10,13]. Few studies regarding melanin deposition have been reported.

To explore the molecular regulation mechanism of Slc7a11 in the melanin deposition of Rex rabbit fur, rabbit melanocytes were isolated and identified. The expression pattern of Slc7a11 in Rex rabbits with different fur colors was analyzed. Furthermore, we verified that POU2F1 has an important regulatory role in the transcriptional activation of the $S l c 7 a 11$ gene promoter. This result provides a theoretical basis for further analysis of the deposition mechanism of the fur pigmentation as well as for the transformation of fur color in animals.

\section{Results}

\subsection{Separation and Identification of Rabbit Melanocytes}

The back skin of black Rex rabbits was collected and cells separated by a two-step enzyme digestion method. After $12 \mathrm{~h}$ of isolation, the keratinocytes were found to have a cobblestone-like appearance and accounted for the majority of cells. The melanocytes, which had the unique bi-polar dendritic morphology, were small in number. However, as cells grew, the keratinocytes gradually died out, the melanocytes continued to divide, and the cell culture became consistent in appearance. When the cells were passed to the third generation, the keratinocytes were almost absent. The melanocytes were dominant with special growing follicles and strong refraction (Figure 1a). 
(a)
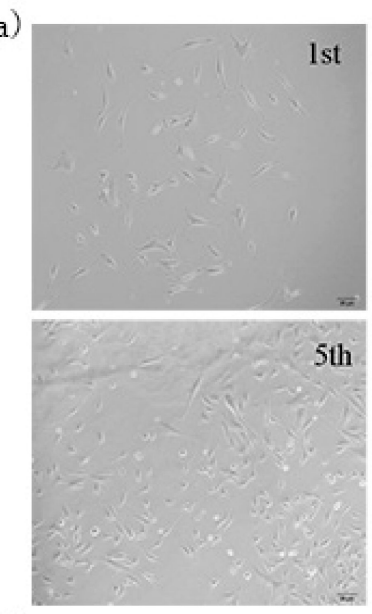

(c)

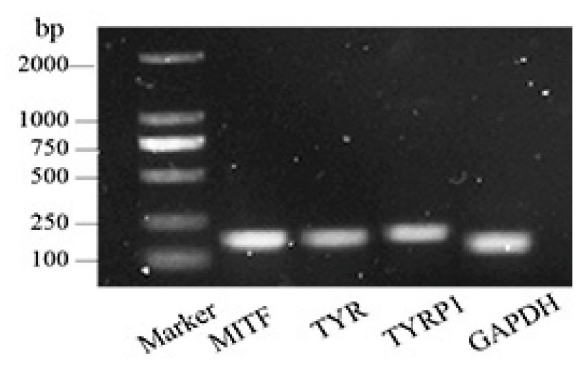

(b)

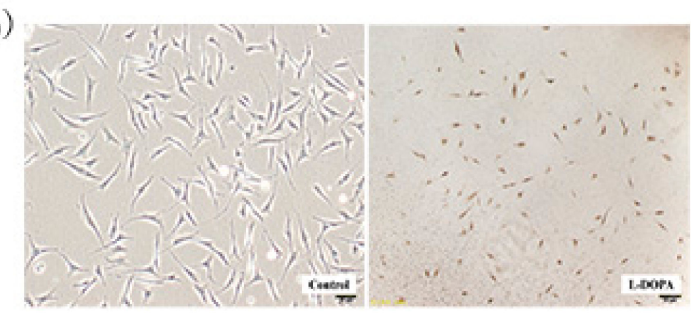

th

(d)
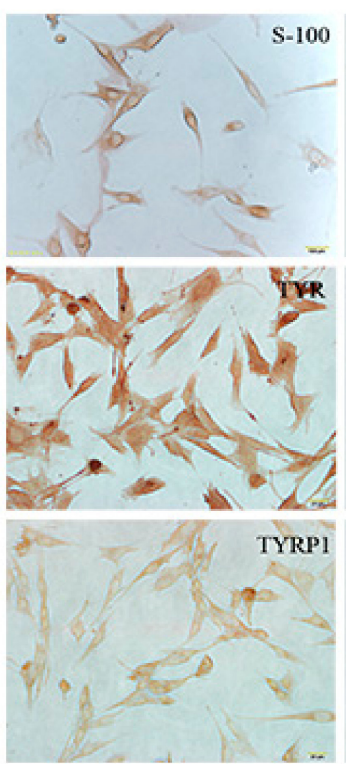

S-100 Control

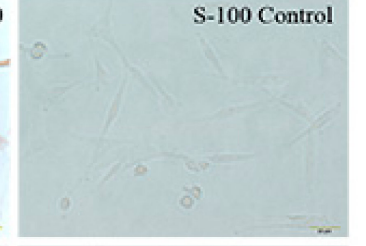

TYR Control

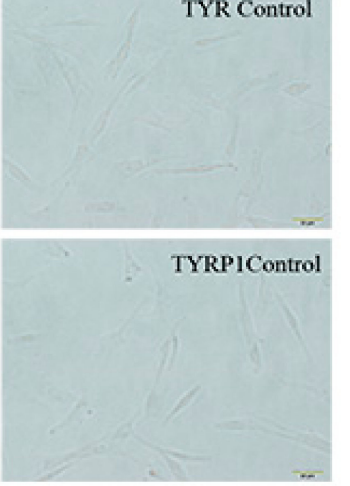

Figure 1. The separation and identification of melanocytes of rabbits. (a) Morphology of the 1st, 3rd, 5th, and 7th generation melanocytes isolated by the two-step enzyme digestion method (100x). After $12 \mathrm{~h}$ of isolation and culture, cobblestone-like keratinocytes and bi-polar dendritic melanocytes were observed. As the cells grew, the keratinocytes gradually metastasized while the melanocytes continued to divide, and the cell culture became more pure. (b) Identification of isolated rabbit melanocytes by L-DOPA staining. The 3rd generation melanocytes were treated with L-DOPA to detect the distribution of brown or black particles in isolated cells $(40 \times)$. Control was treated with PBS (100x). (c) Real-time PCR was used to detect the expression of melanocyte-specific genes such as MITF, TYR, and TYRP1 in isolated cells. (d) Isolated rabbit melanocytes were identified by immunocytochemical staining $(100 \times)$ using melanocyte-specific marker proteins S-100, TYR, and TYRP1 to analyze the expression pattern of these three proteins in the isolated cells.

The melanocyte marker genes MITF, TYR, and TYRP1 were detected by semi-quantitative PCR (Figure $1 b$ ). The isolated cells stained with L-DOPA staining contained brown or black particles (Figure 1c). Immunocytochemical staining of S-100, TYR and TYRP1 revealed that these markers were expressed in melanocytes. Compared with the negative control, S-100 staining showed the cytoplasm and dendrites were positively stained brown. The nucleus was brownish yellow in the TYR staining, and light brown in the TYRP1 staining (Figure 1d). This indicated that the rabbit melanocytes were successfully isolated and identified, providing experimental materials for this study.

\subsection{Analysis of Slc7a11 Gene Expression in Rex Rabbit Skin with Different Fur Colors}

The Slc7a11 cDNA sequence, including 31 bp 5'UTR, 1509 bp open reading frame (ORF), and $132 \mathrm{bp}$ 3'UTRs (poly-[A] tail included), was obtained using RACE and cloning techniques and submitted to GenBank (Accession number KY971639.1) (Figure 2a). The localization of the Slc7a11 protein in the skin tissue of Rex Rabbits was determined by immunohistochemistry. Blue positive reactions were detected in the epidermis, hair bulbs, and hair root-sheaths, with different shades of color, suggesting Slc7a11 was widely expressed (Figure 2b). It was found that the expression level of the Slc7a11 gene 
was highest in skin with PY color, which was 3.7 times that seen in WH. The differences between PY and $\mathrm{WH}$, as well as between PC and WH, were significant $(p<0.01)$ (Figure $2 \mathrm{c}$ ). Wes system analysis showed that the Slc7a11 protein was expressed in all skin tissues. The protein expression level was the highest in PY skin, and the lowest in the WH (Figure 2d,e). The mRNA and protein expression levels of Slc7a11 in Rex rabbit skin with different fur colors showed a significant positive correlation $(\mathrm{R}=0.874, p<0.05)$.

(a)

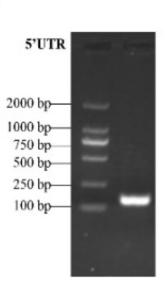

(b)
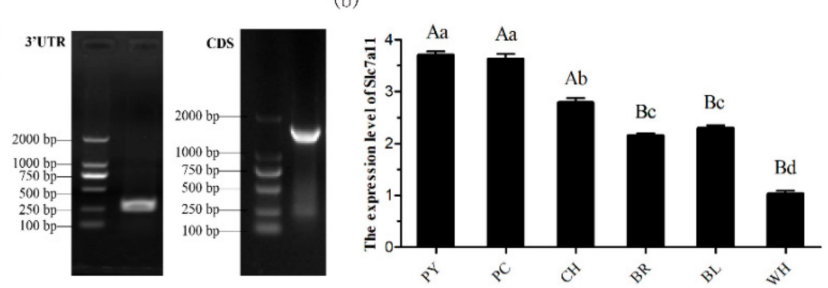

(c)

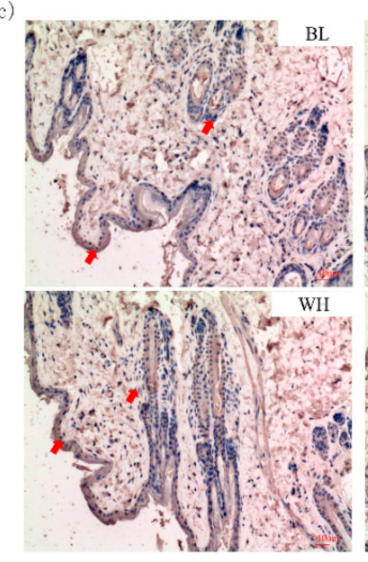

(d)
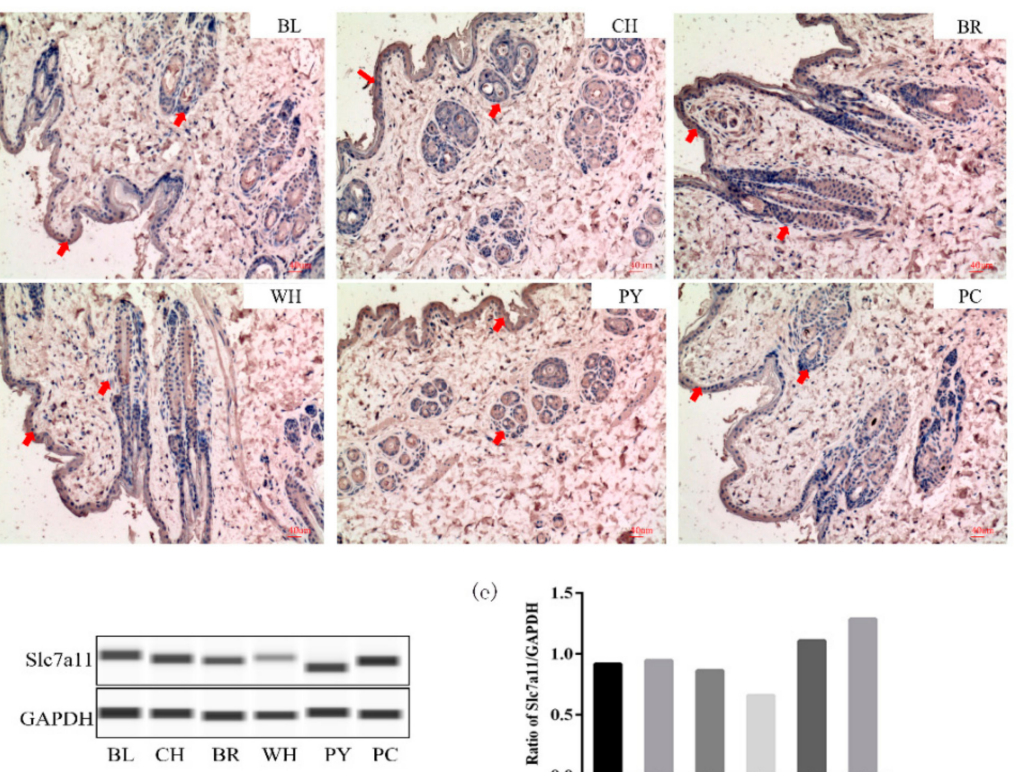

(c)

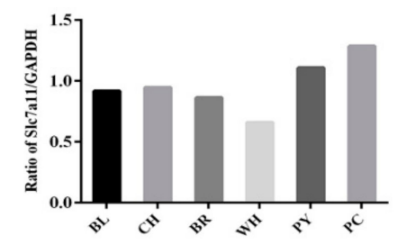

Figure 2. Cloning of rabbit Slc7a11 gene and its expression in Rex Rabbit coat with different fur colors. (a) Full length sequence of rabbit Slc7a11 cDNA was obtained using the RACE technique. The 5'UTR and $3^{\prime}$ UTR sequences were obtained by $5^{\prime}$ RACE and 3' RACE, respectively, and the DNASTAR program was used to assemble the sequence as well as remove redundant sequences to obtain the Rex Rabbit Slc7a11 cDNA sequence. (b) mRNA expression level of Slc7a11 gene in skin tissues of Rex Rabbits with different fur colors by Real-time PCR. Values with different capital letter superscripts signify an extremly significant difference $(p<0.01)$, and values with different small letter superscripts signify a significant difference $(p<0.05)$. (c) Localization of Slc7a11 in the skin of Rex Rabbits with different fur color using immunohistochemical staining. Arrows indicate positive expression of Slc7a11 in the epidermis and hair follicles (×100). (d) The expression level of Slc7a11 (xCT) in skin tissues of Rex Rabbits with different fur colors by the Wes method. (e) The results were analyzed using the Compass program and the relative expression ratio of Slc7a11 was calculated.

\subsection{Effect of Slc7a11 Gene Expression on Melanin Deposition}

In order to further analyze the mechanism of the Slc7a11 gene in melanogenesis, Slc7a11 siRNA interference and overexpression were performed in melanocytes, and qRT-PCR and Wes were used to detect mRNA and protein expression levels of pigment-related genes such as MITF and TYR. The results showed that siRNA-2 and siRNA-3 interferences were significantly lower than that of the blank group $(p<0.05)$, and siRNA-3 had the best effect (Figure 3a,b). pEGFP-N1-Slc7a11 was 
expressed in melanocytes and the expression of Slc7a11 was significantly increased in these cells $(p<0.01)$ (Figure $4 \mathrm{a}, \mathrm{b})$.
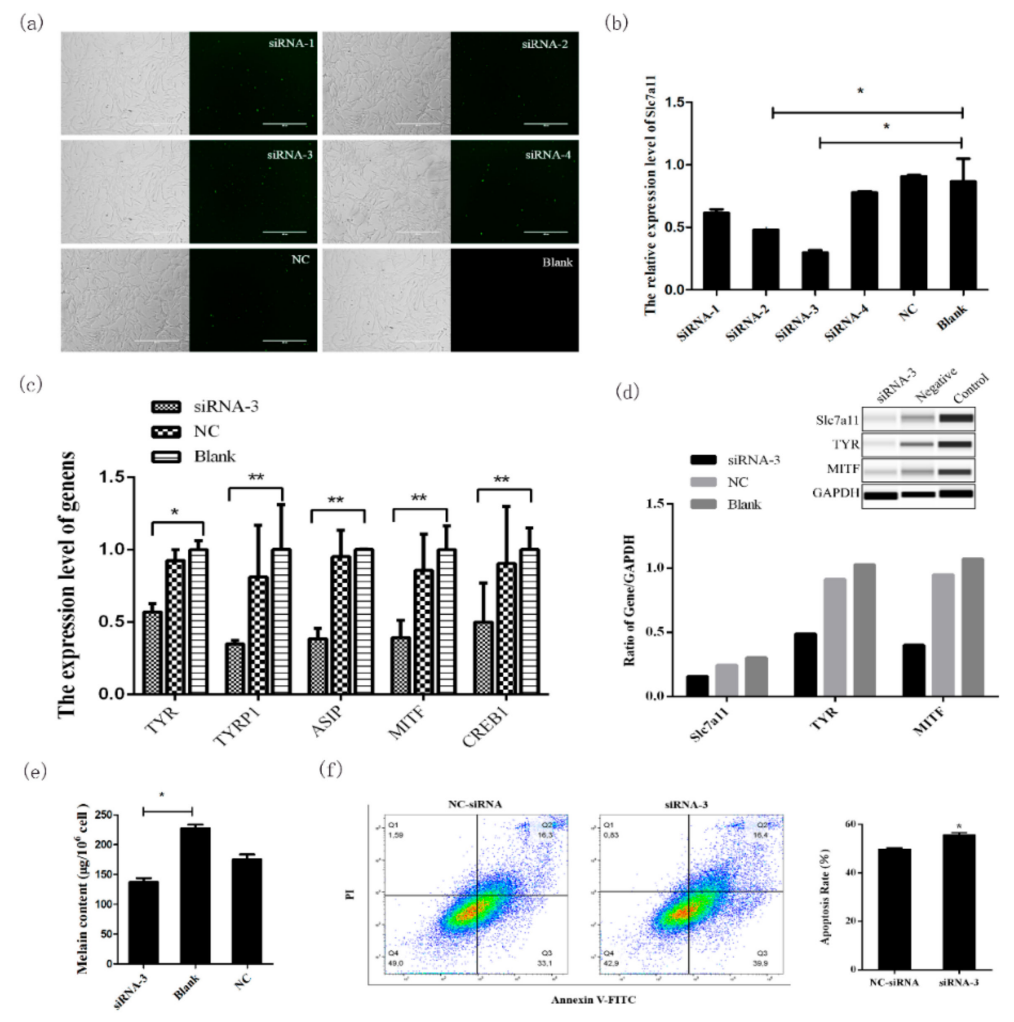

Figure 3. Melanogenesis-related gene expression and melanogenesis were inhibited by Slc7a11 knockdown. An extremly significant difference was signified with "*** $(p<0.01)$, and a significant difference was signified with "** ( $p<0.05)$. (a) Cell morphology $24 \mathrm{~h}$ after transfection of melanocytes by FAM-siRNA. Melanocytes in the logarithmic growth phase were transfected and the transfection was detected by the observation of green fluorescence. (b) Real-time PCR detection of Slc7a11 mRNA expression after siRNA interference. The best siRNA was screened for subsequent experiments. (c) Effects of Slc7a11 interference on the expression of pigmentation-related genes such as MITF, TYR, TYRP1, CREB1, and ASIP. (d) Detection of the expressions of MITF, TYR, and Slc7a11 (xCT) proteins in melanocytes by Wes. The relative expression levels of MITF, TYR, and Slc7a11 (xCT) proteins were calculated and analyzed by the Compass program. (e) The effect of Slc7a11 knockdown on melanogenesis in melanocytes. Melanocytes were collected after siRNA-3 transfection and the melanin level was measured using a microplate reader. (f) Melanocyte apoptosis rate was determined after knockdown of Slc7a11. 
(a)

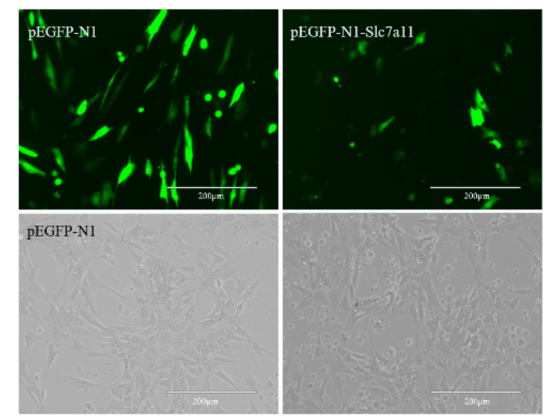

(c)

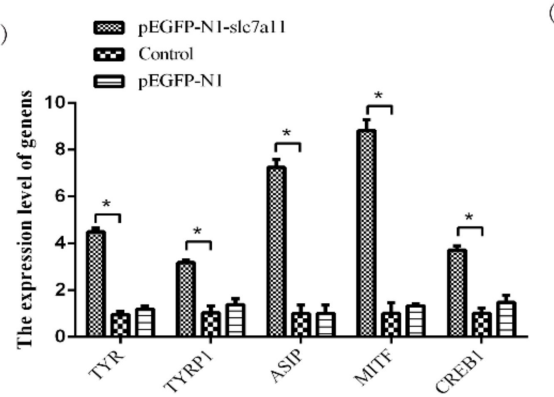

(b)

(d)

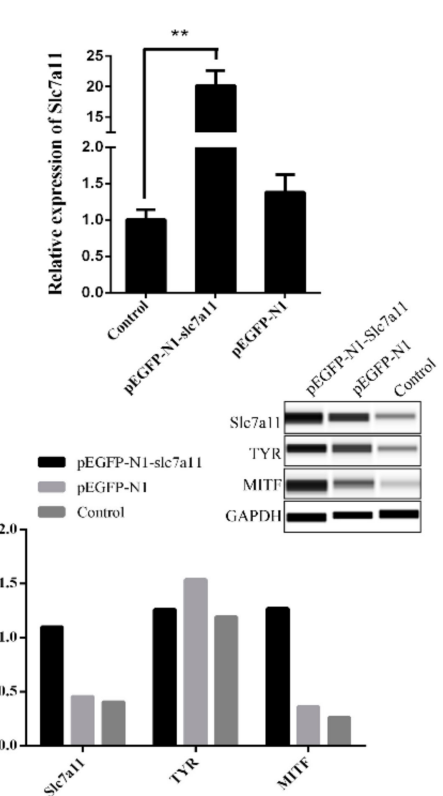

(e)

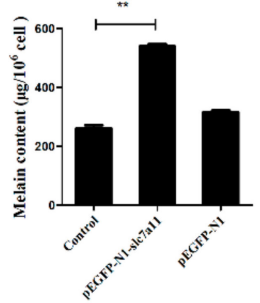

(1)

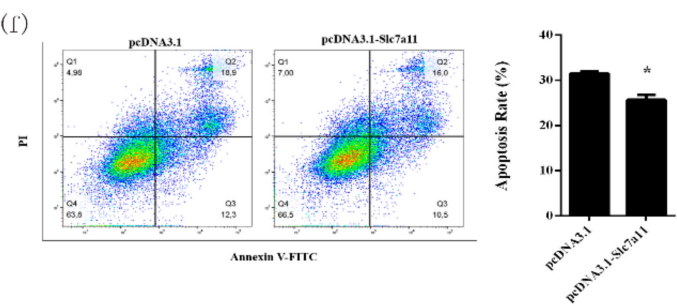

Figure 4. The expression of melanogenesis-related genes and melanogenesis were increased by the overexpression of Slc7a11. An extremly significant difference was signified with "***" $(p<0.01)$, and a significant difference was signified with "**" $(p<0.05)$. (a) Fluorescence detection results of pEGFP-N1-Slc7a11 transfected melanocytes. pEGFP-N1 was used as a control. (b) Detection of the overexpression of Slc7a11 in melanocytes by Real-time PCR. (c) The effect of Slc7a11 overexpression on the expression of pigmentation-related genes such as MITF, TYR, TYRP1, CREB1, and ASIP. (d) Detection of the expressions of MITF, TYR, and Slc7a11 (xCT) proteins in melanocytes by Wes. The relative expression levels of MITF, TYR, and Slc7a11 (xCT) proteins were calculated and analyzed using the Compass program. (e) The effect of Slc7a11 overexpression on melanogenesis in melanocytes. Melanocytes were collected after siRNA-3 transfection and the melanin level was measured using a microplate reader. (f) Melanocyte apoptosis rate was determined after overexpression of Slc7a11.

When Slc7a11 was overexpressed or inhibited, the mRNA (Figures $3 \mathrm{c}$ and $4 \mathrm{c}$ ) and protein expression levels (Figures $3 \mathrm{~d}$ and $4 \mathrm{~d}$ ) of genes involved in the melanogenesis pathways (such as MITF, $T Y R, T Y R P 1, C R E B 1$, and ASIP) also changed significantly. There was a significant positive correlation between mRNA and protein expression $(p<0.05)$, which was consistent with the changes in the expression of Slc7a11. Compared with the control group, the melanin level increased when Slc7a11 was overexpressed; whereas when Slc7a11 was inhibited melanin level decreased (Figures 3e and 4e). The results suggested that $S l c 7 a 11$ affects the expression of pigmentation-related genes such as TYR and MITF, and thus affects melanogenesis by melanocytes. We examined the apoptosis rate in melanocytes after transfecting these cells with siRNA-Slc7a11 and pcDNA3.1-Slc7a11 and found that Slc7a11 decreased melanocyte apoptosis, but inhibition of Slc7a11 enhanced apoptosis (Figures $3 \mathrm{f}$ and $4 \mathrm{f}$ ). 


\subsection{Identification of the Core Region of the Slc7a11 Promoter and Key Transcription Factor POU2F1}

In order to further reveal the regulatory mechanism of the Slc7a11 gene, the promoter sequence $2499 \mathrm{bp}$ before the start codon of Slc7a11 was cloned. Firstly, by predictive analysis of potential transcription factors in the Slc7a11 promoter region, four deletion vectors (P1-P4) were constructed. Dual-luciferase assays showed that the activities of P2 and P3 were comparable ( $p>0.05)$. P2 activity was significantly lower than that of $\mathrm{P} 1$, and the activity of $\mathrm{P} 4$ was significantly lower than that of P3 $(p<0.05)$, indicating that the deletion of $-969 \sim-469$ bp and $-2469 \sim-1969$ bp decreased the activity significantly. The results suggested that there were two active regions in the Slc7a11 promoter, $-969 \sim-469$ bp and $-2469 \sim-1969$ bp, respectively (Figure 5a).

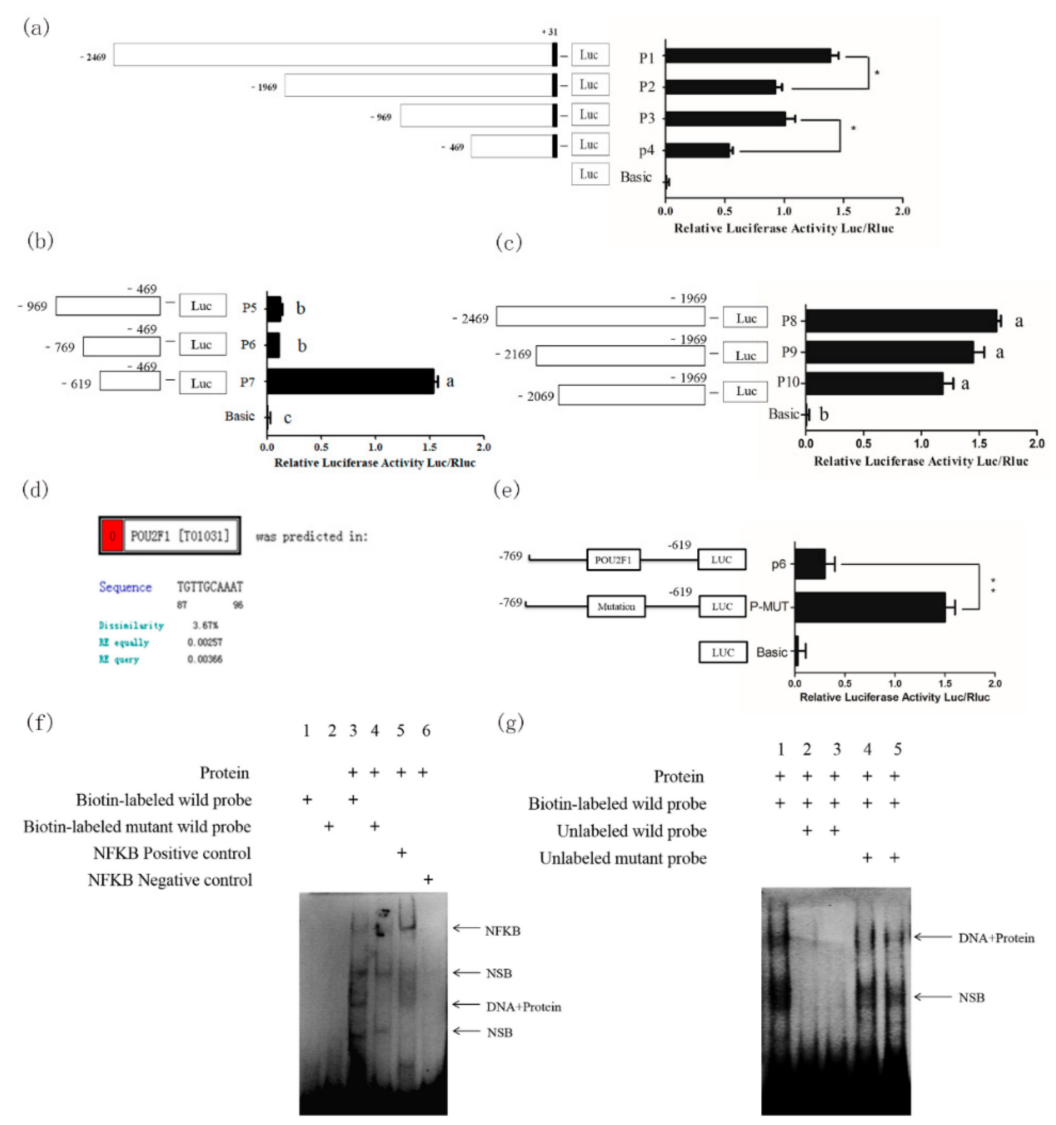

Figure 5. Regulation of transcriptional factor POU2F1 on Slc7a11 promoter activity. An extremly significant difference was signified with "***" $(p<0.01)$, and a significant difference was signified with “*” ( $p<0.05)$. (a) Preliminary analysis of the activity of Slc7a11 promoter-deleted vector series. P1 P4 were constructed and the activity of each fragment was detected using dual luciferase. It was presumed that the Slc7a11 promoter contained two active regions, namely -969 -469 bp and -2469 -1969 bp. (b) Activity detection of a series of vectors with deletions in the $-969 \sim-469$ bp active region of Slc7a11 promoter. P5-P7 were designed and constructed for this region. (c) Activity detection of a series of vectors with deletions in the -2469 - 1969 bp active region of Slc7a11 promoter. P8-P10 were designed and constructed for this region. (d) Prediction of the transcriptional binding site in the Slc7a11 promoter region. The $-769 \sim-619 b p$ region was the primary target based on the results of $(\mathbf{a}-\mathbf{c})$. The results also suggested that transcriptional repressors may be present in this region. The potential transcription factor binding sites were analyzed using the online program PROMO. (e) Site-directed mutagenesis analysis of POU2F1. Based on the predicted position given by PROMO, the POU2F1 binding site was effectively mutated by site-directed mutagenesis and detected by dual-luciferase assay. (f) EMSA suggests that POU2F1 binds to the Slc7a11 core promoter region. The 1st and 2nd lanes were normal and blank mutant probes, respectively, and no bands indicated good probes. The $3 \mathrm{rd}$ and 4 th lanes were biotin-labeled normal and mutation probes, respectively. 
The 5th and 6th lanes were NF-KB positive and negative controls, respectively. NSB stands for non-specific binding. (g) Specific binding of POU2F1 to the Slc7a11 core promoter region using competitive EMSA experiments. In the 2nd and 4th lanes, unlabeled probes were added at a 40:1 ratio to the labeled probes, and in 3rd and 5th lanes unlabeled probes were added at an 80:1 ratio to the labeled probes.

In order to further identify the core transcription factor binding region, a series of deletion vectors were constructed targeting the $-2469 \sim-1969$ bp and -969 -469 bp fragments: P8, P9, P10 for the $-2469 \sim-1969$ bp region, and P5, P6, and P7 for the $-969 \sim-469$ bp region. The activities of P8, P9, and P10 were similar by luciferase assay $(p>0.05)$ (Figure $5 b)$. However, the activities of P5 and P6 were comparable but significantly lower than that of $\mathrm{P} 7$, indicating that the activity was significantly reduced with the deletion of the -769 to $-619 \mathrm{bp}$ region $(p<0.05)$ (Figure $5 \mathrm{c}$ ), suggesting that $-769 \sim-619 \mathrm{bp}$ is the core transcription region of Slc7a11. The predicted POU2F1 binding site ( -713 to $-703 \mathrm{bp}$ ) was found in the -769 to $-619 \mathrm{bp}$ region (Figure $5 \mathrm{~d}$ ). It was found that the promoter activity of Slc7a11 was significantly increased after site-directed mutagenesis $(p<0.01)$, indicating that POU2F1 inhibited the promoter activity of Slc7a11 (Figure 5e).

To further determine whether POU2F1 binds to this site of the Slc7a11 promoter, an EMSA experiment was performed using the nuclear protein of melanocytes (Figure $5 \mathrm{f}$ ). The $3 \mathrm{rd}$ lane showed that the biotin-labeled probe for POU2F1 could bind to the nuclear protein to form a complex band. No band in the 4th lane suggested that mutated POU2F1 could not bind to a nuclear protein to form a complex. The results together revealed that POU2F1 could bind to the Slc7a11 core promoter region, and a competitive EMSA experiment was performed to further determine whether the binding was specific (Figure 5g). A complex band in Lane 1 indicated that the probe was able to bind to the nuclear protein. The 2nd and 3rd lanes were cold-competitive reactions with unlabeled normal probes. No bands were observed, indicating that the unlabeled normal probes were competitively bound to the nuclear protein due to their large amounts, meaning the biotin-labeled probe could hardly bind to the protein. The 4 th and 5 th lanes were cold-competitive groups with unlabeled mutant probes. The unlabeled normal probe did not bind to the protein after mutation, and thus did not compete with the biotin-labeled normal probe, producing protein-probe complex bands. The results confirmed that the POU2F1 protein could bind to the -713 to $-703 \mathrm{bp}$ region and inhibit the activity of the Slc7a11 promoter.

\section{Discussion}

Skin melanocytes are located on the basement membrane between the epidermis and the dermis. Skin melanocytes have been isolated from humans, pigs, and other animals using $0.25 \%$ trypsin and successfully cultured $[14,15]$. In a preliminary study, the back skin of rabbits was digested with trypsin, but the results were not satisfactory, indicating that the use of trypsin alone did not result in sufficient digestion or separation of melanocytes from the skin of hairy animals. However, the use of Dispase II enzyme digestion, allowed us to obtain pure melanocytes for the first time, which laid the foundation for our later work.

Fur color is controlled by different genes in the process of pigment biosynthesis. The differences in color are mainly due to the different ratios between pheomelanin (red and yellow) and eumelanin (black) [16-18]. The main model currently proposed is that the ratio of eumelanin/pheomelanin in mammalian pigments is solely or indirectly regulated by the activity of tyrosinase - the rate-limiting enzyme of melanin synthesis [19]. This model inferred that in the presence of low concentrations of tyrosinase, dopaquinone reacts with cysteine to produce cysteine dopa, thereby increasing the level of pheomelanin [20]. However, this pattern is not yet fully understood.

Studies have confirmed that the $\mathrm{xCT}$ transporter encoded by the Slc7a11 gene is crucial for the regulation of pigments and can directly affect the increase of pheomelanin [9]. Based on previous studies, Rex Rabbits with a variety of natural fur colors were used to explore the expression pattern of 
Slc7a11 gene in dorsal skin tissues with different fur colors. $\mathrm{xCT}$ was expressed in the epidermis, hair bulb, and hair root-sheaths of the skin tissues examined by immunohistochemistry. It is known that melanocytes in the skin are often distributed in different regions when matured and that the only place where melanin can be supplied to the hair shaft is the hair bulb [21-23]. In this study, it was found that the expression sites of the Slc7a11 gene were consistent with the distribution of melanin, suggesting that the protein was related to the formation of Rex Rabbit fur color. Moreover, the Slc7a11 gene had the highest expression level in protein yellow coat, and the lowest level in white coat, by real-time quantitative and Wes system analyses. It is speculated that Slc7a11 affects the formation of cystine, which is reduced to cysteine, and thus alters the production of pheomelanin. This is consistent with studies on alpaca and sheep [24,25].

Knockdown and overexpression analyses of Slc7a11 confirmed that this gene can affect the expression of TYR, MITF, TYRP1, and ASIP in the melanogenesis pathway [3,26]. TYR is a key enzyme in melanin formation and its expression level and activity determine the rate and yield of melanin production. Upon activation, TYR catalyzes the hydroxylation of tyrosine to L-3,4-phenylalanine (DOPA), which is rapidly oxidized to form dopaquinone [27]. MITF regulates the expression of the tyrosine gene family and participates in melanin production [28]. Studies have shown that the expression of TYRP1, ASIP and CREB1 determines skin melanin deposition [29-31]. The Slc7a11 gene affects the transcription of genes involved in melanogenesis, which is closely related to the formation of fur color. Moreover, Slc7a11 decreases melanocyte apoptosis and further affects melanogenesis of melanocytes. These results confirmed that $S l c 7 a 11$ is closely related to the formation of Rex rabbit fur color. The regulatory factors required for such expression patterns would be the next research objective.

Chintala et al. found that the mouse light gray (sut) mutation was due to the inhibition of phaeomelanin production. It was caused by a large deletion of the Slc7a11 gene starting from the 11th intron crossing the 12th exon into the region adjacent to the Pcdh18 gene. This resulted in a change in the $3^{\prime}$ end of Slc7a11 transcription [9]. Based on these results, the identification of similar deletions in the natural populations of Rex Rabbits with six fur colors was carried out. Unfortunately, no such large fragment deletions were found in similar areas. Exon scanning did not show any SNPs sites, indicating that $S l c 7 a 11$ is relatively conserved in the population. Similar results have been seen in humans and sheep $[25,32,33]$. The regulatory mechanism of Slc7a11 is unknown.

Furthermore, the promoter series deletion vector dual-luciferase was used to search for the -769 to $-619 \mathrm{bp}$ transcription core region of Slc7a11. We also confirmed that POU2F1 protein binds to the -713 to -703 bp region of the Slc7a11 promoter to inhibit its activity. POU2F1, also known as Octamer Transcription factor-1 (Oct-1), is a widely expressed POU protein factor. Recent studies suggest that it can regulate target genes associated with processes such as oxidation, anti-cytotoxicity, stem cell function, and cancer development, etc. $[34,35]$. Ethanol has been reported to increase the expression of Slc7a11 by reducing the binding of POU2F1 to the Slc7a11 gene promoter [36]. In this study, POU2F1 was found to specifically bind to the Slc7a11 promoter and inhibit its transcription. Together with the previous finding that Slc7a11 promotes melanin cytochrome deposition, POU2F1 can be used as a target for artificial modification of animal fur colors.

\section{Materials and Methods}

\subsection{Primary Separation and Culture of Rabbit Melanocytes}

Rabbits were injected with anesthetic on the back, and a piece of the back skin $(1.5 \mathrm{~cm} \times 1.5 \mathrm{~cm})$ was dissected. Any particles on the skin surface and subcutaneous connective tissue were removed. After tissue collection, the wounds were treated with iodine tincture. After the experiment, rabbits were anesthetized by an intraperitoneal injection of sodium pentobarbital $(50 \mathrm{mg} / \mathrm{kg})$.

The skin sample was digested with $0.25 \%$ DispaseII enzyme digestion solution (Sigma, Darmstadt, Germany) for $14-16 \mathrm{~h}$ at $4{ }^{\circ} \mathrm{C}$. The epidermis was gently peeled off from the dermis, cut into small pieces, and digested with $0.25 \%$ trypsin (Gibco, Carlsbad, CA, USA) for 8 min at $37^{\circ} \mathrm{C}$. 
The sample was then filtered through a 200 mesh filter and the supernatant discarded. The cells were resuspended in M254 medium (Gibco, Carlsbad, CA, USA) and incubated at $37^{\circ} \mathrm{C}$ in a $5 \% \mathrm{CO}_{2}$ incubator. The cells were digested with $1 \mathrm{~mL}$ of $0.25 \%$ trypsin and subcultured.

This study was carried out in accordance with the recommendations of Animal Care and Use Committee at Yangzhou University. The protocol was approved by the Animal Care and Use Committee at Yangzhou University (Yangzhou, China, 24 October 2017, No. 201710001).

\subsection{DOPA Staining}

Melanocytes in the logarithmic growth phase were used to prepare sterile cell culture slides. The inoculated 24-well plates were cultured for 3 days and treated with $1 \mathrm{~mL}$ of $4 \%$ paraformaldehyde fixative (Solarbio, Beijing, Tongzhou, China) for $30 \mathrm{~min}$ at $4{ }^{\circ} \mathrm{C}$. The plates were washed 3 times with pre-cooled PBS prior to the addition of L-DOPA (Sigma). After incubation in L-DOPA for 1 day, the incubation solution was renewed and the plates were further incubated at $37^{\circ} \mathrm{C}$ for $12 \mathrm{~h}$, with constant observation once every $30 \mathrm{~min}$. The plates were washed with PBS once the staining was complete and observed under a microscope [37].

\subsection{Immunostaining}

Melanocytes in the logarithmic growth phase or skin tissues from Rex rabbits of 6 different fur colors were used to prepare slides. The slides were incubated with primary antibodies Slc7a11 (1:500 rabbit polyclonal, Abcam, Cambridge, UK), S-100 (1:500 mouse monoclonal, Boster, Wuhan, China), TYRP1 (1:250 rabbit polyclonal, Abcam), TYR (1:1000 rabbit polyclonal, Abcam) overnight at $4{ }^{\circ} \mathrm{C}$, with PBS as a negative control. The slides were subsequently incubated with IgG secondary antibody (1:2500 goat polyclonal, Abcam) at $37^{\circ} \mathrm{C}$ for $20 \mathrm{~min}$ and developed for $3-5 \mathrm{~min}$ at room temperature in the dark with freshly prepared DAB solution (Boster). The slides were observed under a microscope.

\subsection{RACE and Cloning of Slc7a11 Gene}

Three specific 5' RACE primers and two 3' RACE primers were designed according to the Race kit instructions (Invitrogen \& Clontech, Carlsbad, CA, USA) (Table 1). The full-length cDNA sequence of the Slc7a11 gene was assembled based on known sequences and $5^{\prime}$ and $3^{\prime}$ RACE results, and submitted to NCBI (Accession no.: KY971639.1). The Slc7a11 cDNA was reconstructed into the pEGFP-N1 vector with restriction enzymes HindIII and SacII.

Table 1. Primers used for Slc7a11 RACE and cloning.

\begin{tabular}{ccc}
\hline Name & Sequence $\mathbf{( 5}^{\prime}$ to $\mathbf{3}^{\prime} \mathbf{)}$ & Experiment \\
\hline GSP1 & CACTTTCTCCTGCCCA & $5^{\prime}$ RACE \\
GSP2 & GGCTCCTTGCCACCCAT & $5^{\prime}$ RACE \\
GSP3 & TCCCGTTCACGTTTCCC & $5^{\prime}$ RACE \\
C048-1 & CCCTTTCCCTTTATTCGGACCCAT & $3^{\prime}$ RACE \\
C048-2 & CGGGGTCCCTGCCTACTATCTCTT & $3^{\prime}$ RACE \\
\hline Slc7a11 CDS-F & ATGGTCAGAAAACCTGTTGTGTCCACC & CDS amplification \\
Slc7a11 CDS-R & TTATTTACGACAGTCTTCTTCAGGTACA & \\
Slc7a11-F & CCCAAGCTTATGGTCAGAAAACCTGTTGTGTCCACC & \multirow{2}{*}{ Vector construction } \\
\hline \multicolumn{2}{c}{ Note: Bold letters indicate restriction enzyme cutting sites, italics indicate protective base. }
\end{tabular}

\subsection{Knockdown of Slc7a11 by siRNA}

Fluorescently labeled siRNAs (with 5' FAM modification) and Negative Control siRNAs were purchased from Shanghai GenePharma Co., Ltd (Table 2). When melanocyte confluence reached about $65 \%$, the siRNA oligo/Lipofectamine ${ }^{\mathrm{TM}} 2000$ (Invitrogen, Carlsbad, CA, USA) complex at a 
ratio of 1:2 was prepared for transfection. After $24 \mathrm{~h}$, the transfection efficiency was examined by fluorescence microscopy.

Table 2. The primer sequences of Slc7a11 siRNA primers.

\begin{tabular}{ccc}
\hline Name & F/R & Sequence (5' $^{\prime}$ to $\mathbf{3}^{\prime}$ ) \\
\hline \multirow{2}{*}{ Negative Control } & $\mathrm{F}$ & UUCUCCGAACGUGUCACGUTT \\
& $\mathrm{R}$ & ACGUGACACGUUCGGAGAATT \\
\hline \multirow{2}{*}{ siRNA-1 } & F1 & CCAUUAUCAUUGGCACCAUTT \\
& R1 & AUGGUGCCAAUGAUAAUGGTT \\
\hline \multirow{2}{*}{ siRNA-2 } & F2 & GCAGCGACUGCUGUGAUAUTT \\
& R2 & AUAUCACAGCAGUCGCUGCTT \\
\hline \multirow{2}{*}{ siRNA-3 } & F3 & GCAGUGAUGGUCCUAAAUATT \\
& R3 & UAUUUAGGACCAUCACUGCTT \\
\hline \multirow{2}{*}{ siRNA-4 } & F4 & CCAUGAUUCAUGUCCGCAATT \\
& R4 & UUGCGGACAUGAAUCAUGGTT \\
\hline
\end{tabular}

\subsection{Real-Time PCR}

Real-time PCR was carried out using SYBR ${ }^{\circledR}$ Premix Ex Taq ${ }^{\mathrm{TM}}$ II (TaKaRa, Dalian, China) on an Applied Bio systems 7500 Real-Time PCR System with the following program: 1 cycle at $95^{\circ} \mathrm{C}$ for $30 \mathrm{~s}$, followed by 40 cycles of $95^{\circ} \mathrm{C}$ for $5 \mathrm{~s}$, and $60^{\circ} \mathrm{C}$ for $30 \mathrm{~s}$. The primers used for detection of gene expression are shown in Table 3. Each sample was measured three times, and the results were normalized to GAPDH. The relative expression of the target gene was calculated by the $\Delta \Delta \mathrm{Ct}$ method; namely, the fold difference between the target gene and the reference gene (experimental group)/the fold difference

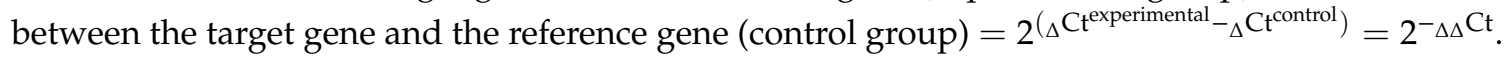

Table 3. Primer sequences used for real-time PCR.

\begin{tabular}{|c|c|c|c|}
\hline Name & Sequence $\left(5^{\prime}\right.$ to $\left.3^{\prime}\right)$ & Product Length/bp & $\mathrm{Tm} /{ }^{\circ} \mathrm{C}$ \\
\hline Slc7a11-RT-F & TCACCATTGGCTACGTGCT & \multirow[b]{2}{*}{142} & \multirow[b]{2}{*}{60} \\
\hline Slc7a11-RT-R & GCCACAAAGATCGGAACTGCT & & \\
\hline GAPDH-RT-F & CACCAGGGCTGCTTTTAACTCT & \multirow{2}{*}{155} & \multirow{2}{*}{60} \\
\hline GAPDH-RT-R & CTTCCCGTTCTCAGCCTTGACC & & \\
\hline CREB1-RT-F & ССТССССАGСАСТТССТАСАСА & \multirow{2}{*}{158} & \multirow{2}{*}{58} \\
\hline CREB1-RT-R & TTCAGCTCCTCAATCAGCGTCT & & \\
\hline TYR-RT-F & ATTTTCCTCGAGCCTGTACCTCC & \multirow{2}{*}{147} & \multirow{2}{*}{59} \\
\hline TYR-RT-R & GCCAAGACTCCCGTTCATCCAC & & \\
\hline TYRP1-RT-F & СCGTCTTCTCTCAATGGCGAGT & \multirow{2}{*}{154} & \multirow{2}{*}{61} \\
\hline TYRP1-RT-R & TGCACCATCGGTCTAGCCACA & & \\
\hline ASIP-RT-F & CTGTGCTTCCTCACTGCCTATAGCC & \multirow{2}{*}{187} & \multirow{2}{*}{60} \\
\hline ASIP-RT-R & TTCAGCGCCACAATGGAGACCGAA & & \\
\hline MITF-RT-F & AGCTTGCCATGTCCAAACCAG & \multirow{2}{*}{165} & \multirow{2}{*}{58} \\
\hline MITF-RT-R & TTCATACTTGGGCACTCGCTCT & & \\
\hline
\end{tabular}

\subsection{Simple Western Analysis}

Pre-cooled RIPA lysis buffer (Sigma) was mixed with PMSF (with a final concentration of $1 \mathrm{mM}$ ) and added to the tissue or cell samples, which were centrifuged at $10,000 \mathrm{rpm}$ for $5 \mathrm{~min}$ at $4{ }^{\circ} \mathrm{C}$. The supernatant was discarded and the total protein obtained. Simple Western analysis was performed using the Wes Simple Western (Protein Simple) system. This assay uses an automated Western system-no gels, no transfer devices, no blots, no film and no manual analysis, resulting in a digital result. The test results were analyzed using the Compass program. 


\subsection{Apoptosis Assay}

The apoptosis rate was measured with an Annexin V-FITC Apoptosis Detection Kit (Vazyme, Nanjing, China), according to the manufacturer's instructions. After transfection, the cells were collected and washed twice with PBS, then centrifuged at $300 \mathrm{~g}, 4^{\circ} \mathrm{C}$ for $5 \mathrm{~min}$. The cells were resuspended in $100 \mu \mathrm{L} 1 \times$ Binding Buffer and mixed gently followed by the addition of $5 \mu \mathrm{L}$ Annexin V-FITC and $5 \mu \mathrm{L}$ PI Staining Solution, which were allowed to incubate at room temperature for $10 \mathrm{~min}$ in the dark. Finally, cells were added to $400 \mu \mathrm{L} 1 \times$ Binding Buffer and mixed gently. Cells were sorted by fluorescence-activated cell sorting using the Flow cytometer FACSAria SORP (Becton Dickinson, Franklin Lakes, NJ, USA). The apoptosis rate was calculated using the following equation: total number of cells is composed of number of cells in the right upper quadrant and number of cells in the right lower quadrant.

\subsection{Determination of Melanin Level}

The cells were lysed with $1 \mathrm{~mL} 0.2 \mathrm{~mol} / \mathrm{L} \mathrm{NaOH}$. The cell lysate was collected and incubated at $37^{\circ} \mathrm{C}$ for $2 \mathrm{~h}$. Wavelength measurement was performed at $475 \mathrm{~nm}$ using a microplate reader. The standard curve was plotted using the Melanin synthetic standard (Sigma). Each group was repeated 3 times, from which the melanin level was calculated.

\subsection{Luciferase Vector Construction and Reporter Assays}

Promoter-specific primers were designed using O1igo7 (Table 4), and the Slc7a11 promoter region was analyzed using PROMO (http:/www.cbs.dtu.dk/services/Promoter/) to obtain possible transcription factor binding sites. The Slc7a11 promoter was reconstructed into the pGL3-basic vector with restriction enzymes KpnI and BgIII. The internal reference plasmid pRL-TK and the recombinant plasmid were co-transfected into RAB-9 cells (ATCC), with the pGL3-basic plasmid and pRL-TK plasmid co-transfected cells as the negative control group and the cells with no substance transfected as the blank group. The transfected cells were collected and analyzed using the Dual-Luciferase Reporter Assay System (Promega, Madison, WI, USA).

Table 4. Primers used for construction of Slc7a11 promoter deletion plasmids.

\begin{tabular}{|c|c|c|c|}
\hline Name & Sequence $\left(5^{\prime}\right.$ to $\left.3^{\prime}\right)$ & Product Length/bp & Usage \\
\hline P1-F & GGGGTACCACTTTGGGATTGGTGCAG & \multirow[b]{2}{*}{500} & \multirow[b]{2}{*}{ P1 } \\
\hline P1-R & GAAGATCTAGTGATACCAGGGCAAAA & & \\
\hline P2-F & GGGGTACCTTCTTCCACTTAGTCCAG & \multirow{2}{*}{1000} & \multirow{2}{*}{ P2 } \\
\hline P2-R & GAAGATCTGTAGTGATACCAGGGCAA & & \\
\hline P3-F & GGGGTACCTGAAAGTCTGAATTAAACACA & \multirow[t]{2}{*}{2000} & \multirow[t]{2}{*}{ P3 } \\
\hline P3-R & GAAGATCTTACCAGGGCAAAAAGACAACA & & \\
\hline P4-F & GGGGTACCCСTCCCAAGGTACACAGT & \multirow[t]{2}{*}{2500} & \multirow[t]{2}{*}{$\mathrm{P} 4$} \\
\hline P4-R & GAAGATCTAGGGCAAAAAGACAACAC & & \\
\hline P5-F & GGGGTACCAATTTCTTCCACTTAGTCCA & \multirow[t]{2}{*}{500} & \multirow[t]{2}{*}{ P5 } \\
\hline P5-R & GAAGATCTTCAGAACAGACCTTCAGAGA & & \\
\hline P6-F & GGGGTACCGATTTAACTGTGTTGCTAGG & \multirow[t]{2}{*}{300} & \multirow[t]{2}{*}{ P6 } \\
\hline P6-R & GAAGATCTTCCCAAAGTAGTAAATTCAG & & \\
\hline P7-F & GGGGTACCATATTTTTACTTGTGAGTTTAGGAA & \multirow[t]{2}{*}{150} & \multirow[t]{2}{*}{ P7 } \\
\hline P7-R & GAAGATCTTCAGAACAGACCTTCAGAGAGATTG & & \\
\hline P8-F & GGGGTACCTTGCTGCCTCCCAAGGTACAC & \multirow[t]{2}{*}{500} & \multirow[t]{2}{*}{ P8 } \\
\hline P8-R & GAAGATCTCССТCTCTGCСТTTCTCTCTG & & \\
\hline P9-F & GGGGTACCСТTTCСАСССАСАССТАСССТ & \multirow[t]{2}{*}{200} & \multirow[t]{2}{*}{ P9 } \\
\hline P9-R & GAAGATCTCСTCTCTGCСТTTCTCTCTGT & & \\
\hline P10-R & GGGGTACCATATTTAATGGTGTGTGTAA & \multirow[t]{2}{*}{100} & \multirow[t]{2}{*}{$\mathrm{P} 10$} \\
\hline P10-R & GAAGATCTGTGTTTAATTCAGACTTTCA & & \\
\hline
\end{tabular}

Note: Bold letters indicate restriction enzyme cutting sites, italics indicate protective base. 


\subsection{Electrophoresis Mobility Shift Assay (EMSA)}

The nuclear proteins of melanocytes were extracted and the concentrations determined. Based on the binding sequence of POU2F1, normal and mutant probes were designed (Table 5) and biotinylated at the $5^{\prime}$ end. The EMSA reaction system was formulated as shown in Table 6 . The cold-competitive EMSA reaction system is shown in Table 7. The samples were analyzed by Native-PAGE, transferred, and UV cross-linked prior to carrying out the chemiluminescence reaction, development, and photographing.

Table 5. Normal and mutant probes for POU2F1.

\begin{tabular}{cr}
\hline Name & Sequence $\left(\mathbf{5}^{\prime} \mathbf{- 3}^{\prime} \mathbf{)}\right.$ \\
\hline Normal probes & TGCTTGTTGCAAATAGTCTAGCTAGACTATTTGCAACAAGCA \\
Mutant probes & TGCTGTCGCAGATAGTCTAGCTAGACTATCTGCGACAGCA \\
\hline & Note: Bold letters indicate mutation sites.
\end{tabular}

Table 6. EMSA reaction system.

\begin{tabular}{ccccccc}
\hline Ingredient & $\mathbf{1}$ & $\mathbf{2}$ & $\mathbf{3}$ & $\mathbf{4}$ & $\mathbf{5}$ & $\mathbf{6}$ \\
\hline PolydI:dC/ $\mu \mathrm{L}$ & 0.5 & 0.5 & 0.5 & 0.5 & 0.5 & 0.5 \\
$10 \times$ Binding Buffer $/ \mu \mathrm{L}$ & 0.0 & 0.0 & 1.5 & 1.5 & 1.5 & 1.5 \\
Nuclear protein $/ \mu \mathrm{L}$ & 0.0 & 0.0 & 2.0 & 2.0 & 2.0 & 2.0 \\
Biotin-labeled normal probe $/ \mu \mathrm{L}$ & 0.5 & 0.0 & 0.5 & 0.0 & 0.0 & 0.0 \\
Biotin-labeled mutant probe $/ \mu \mathrm{L}$ & 0.0 & 0.5 & 0.0 & 0.5 & 0.0 & 0.0 \\
Positive nuclear extract NF-KB & 0.0 & 0.0 & 0.0 & 0.0 & 0.5 & 0.0 \\
Negative nuclear extract NF-KB & 0.0 & 0.0 & 0.0 & 0.0 & 0.0 & 0.5 \\
ddH $_{2} \mathrm{O} / \mu \mathrm{L}$ & 14.5 & 14.5 & 11.5 & 11.5 & 11 & 11 \\
\hline
\end{tabular}

Table 7. Cold-competitive EMSA reaction system.

\begin{tabular}{cccccc}
\hline Ingredient & $\mathbf{1}$ & $\mathbf{2}$ & $\mathbf{3}$ & $\mathbf{4}$ & $\mathbf{5}$ \\
\hline Unlabeled probe/labeled probe & & $40: 1$ & $80: 1$ & $40: 1$ & $80: 1$ \\
10× Binding Buffer/ $\mu \mathrm{L}$ & 1.5 & 1.5 & 1.5 & 1.5 & 1.5 \\
Nuclear protein $/ \mu \mathrm{L}$ & 2.0 & 2.0 & 2.0 & 2.0 & 2.0 \\
Biotin-labeled normal probe $/ \mu \mathrm{L}$ & 0.5 & 0.5 & 0.5 & 0.5 & 0.5 \\
Unlabeled normal probe & 0.0 & 1.0 & 3.0 & 0.0 & 0.0 \\
Unlabeled mutant probe & 0.0 & 0.0 & 0.0 & 1.0 & 3.0 \\
$\mathrm{dd}_{2} \mathrm{O} / \mu \mathrm{L}$ & 11.0 & 10.0 & 8.0 & 10.0 & 8.0 \\
\hline
\end{tabular}

\subsection{Statistical Analysis}

Each experiment was repeated at least three times. Statistical analysis Q-Q plot was used to check the normality of the data. Statistical significance between experimental and control groups was analyzed by Independent-Sample Test and one-way ANOVA. The results are presented as mean \pm standard deviation (SD) at two levels of significance, $p<0.05$ and $p<0.01$.

\section{Conclusions}

In summary, rabbit melanocytes were isolated and identified. The Slc7a11 expression levels in the protein yellow colored skin tissue was higher than those from other fur colors. It was found that Slc7a11 significantly affected the protein and mRNA expression of TYR and MITF, inhibiting melanocyte apoptosis and affecting melanogenesis by melanocytes. Furthermore, we that POU2F1 regulated the activity of the rabbit Slc7a11 promoter. Our results provide a theoretical basis for further exploration of the role Slc7a11 plays in pigmentation.

Author Contributions: Y.C. conceived and designed the experiments, performed the experiments, wrote the paper. S.H. performed the experiments. L.M. prepared figures and/or tables. B.Z. analyzed the data. M.W. and 
N.Y. contributed reagents/materials/analysis tools. G.B. and C.Z. prepared figures and/or tables. X.W. conceived and designed the experiments, reviewed drafts of the paper.

Funding: This work was supported by the Modern Agricultural Industrial System Special Funding (CARS-43-A-1), National Natural Science Foundation of China (Grant No. 31702081), and Science and Technology Major Project of New Variety Breeding (Livestock and Poultry) of Zhejiang Province, China (2016C02054-10).

Conflicts of Interest: The authors declare no conflict of interest. The funders had no role in the design of the study; in the collection, analyses, or interpretation of data; in the writing of the manuscript, or in the decision to publish the results.

\begin{tabular}{ll}
\multicolumn{2}{l}{ Abbreviations } \\
Slc7a11 & Solute carrier family 7 member 11 \\
POU2F1 & POU domain class 2 transcription factor 1 \\
RNA-Seq & transcriptome sequencing \\
qRT-PCR & quantitative real time PCR \\
BL & black \\
CH & chinchilla \\
WH & white \\
BR & brown \\
PY & protein yellow \\
PC & protein chinchilla \\
EMSA & Electrophoresis mobility shift assay \\
SD & standard deviation \\
ORF & open reading frame \\
PBS & Phosphate buffer solution \\
Oct-1 & Octamer Transcription factor-1
\end{tabular}

\section{References}

1. Slominski, A.; Tobin, D.J.; Shibahara, S.; Wortsman, J. Melanin pigmentation in mammalian skin and its hormonal regulation. Physiol. Rev. 2004, 84, 1155-1228. [CrossRef]

2. Hartman, M.L.; Czyz, M. Mitf in melanoma: Mechanisms behind its expression and activity. Cell. Mol. Life Sci. 2015, 72, 1249-1260. [CrossRef]

3. Hida, T.; Wakamatsu, K.; Sviderskaya, E.V.; Donkin, A.J.; Montoliu, L.; Lamoreux, M.L.; Yu, B.; Millhauser, G.L.; Ito, S.; Barsh, G.S.; et al. Agouti protein, mahogunin, and attractin in pheomelanogenesis and melanoblast-like alteration of melanocytes: A camp-independent pathway. Pigm. Cell Melanoma Res. 2009, 22, 623-634. [CrossRef]

4. Qin, L.Z.; Wang, W.Z.; Shi, L.J.; Wan, X.Y.; Yan, X.R.; Weng, Q.Q.; Wu, X.S. Transcriptome expression profiling of fur color formation in domestic rabbits using solexa sequencing. Genet. Mol. Res. 2016, 15, 1-16. [CrossRef] [PubMed]

5. Ito, S. Encapsulation of a reactive core in neuromelanin. Proc. Natl. Acad. Sci. USA 2006, 103, 14647-14648. [CrossRef] [PubMed]

6. Shih, A.Y.; Murphy, T.H. Xct cystine transporter expression in hek293 cells: Pharmacology and localization. Biochem. Biophys. Res. Commun. 2001, 282, 1132-1137. [CrossRef]

7. Sato, H.; Tamba, M.; Ishii, T.; Bannai, S. Cloning and expression of a plasma membrane cystine/glutamate exchange transporter composed of two distinct proteins. J. Biol. Chem. 1999, 274, 11455-11458. [CrossRef] [PubMed]

8. Kim, J.Y.; Kanai, Y.; Chairoungdua, A.; Cha, S.H.; Matsuo, H.; Kim, D.K.; Inatomi, J.; Sawa, H.; Ida, Y.; Endou, H. Human cystine/glutamate transporter: Cdna cloning and upregulation by oxidative stress in glioma cells. BBA Biomembr. 2001, 1512, 335-344. [CrossRef]

9. Chintala, S.; Li, W.; Lamoreux, M.L.; Ito, S.; Wakamatsu, K.; Sviderskaya, E.V.; Bennett, D.C.; Park, Y.M.; Gahl, W.A.; Huizing, M.; et al. Slc7a11 gene controls production of pheomelanin pigment and proliferation of cultured cells. Proc. Natl. Acad. Sci. USA 2005, 102, 10964-10969. [CrossRef] [PubMed]

10. Liu, R.R.; Brown, C.E.; Murph, T.H. Differential regulation of cell proliferation in neurogenic zones in mice lacking cystine transport by xct. Biochem. Bioph. Res. Commun. 2007, 364, 528-533. [CrossRef] 
11. Bridges, C.C.; Kekuda, R.; Wang, H.P.; Prasad, P.D.; Mehta, P.; Huang, W.; Smith, S.B.; Ganapathy, V. Structure, function, and regulation of human cystine/glutamate transporter in retinal pigment epithelial cells. Investig. Ophth. Vis. Sci. 2001, 42, 47-54.

12. Qin, S.; Colin, C.; Hinners, I.; Gervais, A.; Cheret, C.; Mallat, M. System x(c)(-) and apolipoprotein e expressed by microglia have opposite effects on the neurotoxicity of amyloid-beta peptide 1-40. J. Neurosci. 2006, 26, 3345-3356. [CrossRef]

13. Wu, Y.D.; Sun, X.J.; Song, B.; Qiu, X.L.; Zhao, J.J. Mir-375/slc7a11 axis regulates oral squamous cell carcinoma proliferation and invasion. Cancer Med. 2017, 6, 1686-1697. [CrossRef]

14. Li, L.; Fukunagakalabis, M.; Herlyn, M. Isolation and cultivation of dermal stem cells that differentiate into functional epidermal melanocytes. Methods Mol. Biol. 2012, 806, 15. [PubMed]

15. Jule, S.; Bosse, P.; Egidy, G.; Panthier, J.J. Establishment and characterization of a normal melanocyte cell line derived from pig skin. Pigm. Cell Res. 2003, 16, 407-410. [CrossRef]

16. Barsh, G.; Cotsarelis, G. How hair gets its pigment. Cell 2007, 130, 779-781. [CrossRef]

17. Ito, S.; Wakamatsu, K. Chemistry of mixed melanogenesis - pivotal roles of dopaquinone. Photochem. Photobiol. 2008, 84, 582-592. [CrossRef] [PubMed]

18. Ito, S.; Wakamatsu, K.; Ozeki, H. Chemical analysis of melanins and its application to the study of the regulation of melanogenesis. Pigm. Cell Res. 2000, 13, 103-109. [CrossRef]

19. Wagner, C.A.; Lang, F.; Broer, S. Function and structure of heterodimeric amino acid transporters. Am. J. Physiol. Cell Physiol. 2001, 281, C1077-C1093. [CrossRef]

20. Ito, S. A chemist's view of melanogenesis. Pigm. Cell Res. 2003, 16, 230-236. [CrossRef]

21. Tobin, D.J.; Slominski, A.; Botchkarev, V.; Paus, R. The fate of hair follicle melanocytes during the hair growth cycle. J. Investig. Derm. Symp. Proc. 1999, 4, 323-332. [CrossRef]

22. Tobin, D.J. The cell biology of human hair follicle pigmentation. Pigm. Cell Melanoma Res. 2011, $24,75-88$. [CrossRef] [PubMed]

23. Slominski, A.; Wortsman, J.; Plonka, P.M.; Schallreuter, K.U.; Paus, R.; Tobin, D.J. Hair follicle pigmentation. J. Investig. Derm. 2005, 124, 13-21. [CrossRef] [PubMed]

24. Tian, X.; Meng, X.L.; Wang, L.Y.; Song, Y.F.; Zhang, D.L.; Ji, Y.K.; Li, X.J.; Dong, C.S. Molecular cloning, mrna expression and tissue distribution analysis of slc7a11 gene in alpaca (lama paco) skins associated with different coat colors. Gene 2015, 555, 88-94. [CrossRef]

25. He, X.; Li, H.T.; Zhou, Z.Y.; Zhao, Z.S.; Li, W. Production of brown/yellow patches in the slc7a11 transgenic sheep via testicular injection of transgene. J. Genet. Genom. 2012, 39, 281-285. [CrossRef]

26. Gutierrez-Gil, B.; Wiener, P.; Williams, J.L. Genetic effects on coat colour in cattle: Dilution of eumelanin and phaeomelanin pigments in an $\mathrm{f} 2$-backcross charolais $\mathrm{x}$ holstein population. BMC Genet. 2007, 8, 56. [CrossRef]

27. Fitzpatrick, T.B.; Miyamoto, M.; Ishikawa, K. The evolution of concepts of melanin biology. Arch. Dermatol. 1967, 96, 305-323. [CrossRef]

28. Levy, C.; Khaled, M.; Fisher, D.E. Mitf: Master regulator of melanocyte development and melanoma oncogene. Trends Mol. Med. 2006, 12, 406-414. [CrossRef]

29. Jackson, I.J. A cdna encoding tyrosinase-related protein maps to the brown locus in mouse. Proc. Natl. Acad. Sci. USA 1988, 85, 4392-4396. [CrossRef]

30. Mundy, N.I.; Kelly, J. Investigation of the role of the agouti signaling protein gene (asip) in coat color evolution in primates. Mamm. Genome 2006, 17, 1205-1213. [CrossRef]

31. Yamaguchi, Y.; Hearing, V.J. Physiological factors that regulate skin pigmentation. Biofactors 2009, 35, 193-199. [CrossRef]

32. Gasol, E.; Jimenez-Vidal, M.; Chillaron, J.; Zorzano, A.; Palacin, M. Membrane topology of system x(c)(-) light subunit reveals a re-entrant loop with substrate-restricted accessibility. J. Biol. Chem. 2004, 279, 31228-31236. [CrossRef] [PubMed]

33. Sato, H.; Tamba, M.; Kuriyama-Matsumura, K.; Okuno, S.; Bannai, S. Molecular cloning and expression of human xct, the light chain of amino acid transport system x(c)(-). Antioxid. Redox Signal. 2000, 2, 665-672. [CrossRef]

34. Vazquez-Arreguin, K.; Tantin, D. The oct1 transcription factor and epithelial malignancies: Old protein learns new tricks. BBA Gene Regul. Mech. 2016, 1859, 792-804. [CrossRef] 
35. Maddox, J.; Shakya, A.; South, S.; Shelton, D.; Andersen, J.N.; Chidester, S.; Kang, J.; Gligorich, K.M.; Jones, D.A.; Spangrude, G.J.; et al. Transcription factor oct1 is a somatic and cancer stem cell determinant. PLOS Genet. 2012, 8, e1003048. [CrossRef]

36. Lin, X.H.; Yang, H.; Zhang, H.F.; Zhou, L.C.; Guo, Z.M. A novel transcription mechanism activated by ethanol induction of slc7a11 gene expression via inhibition of the DNA-binding activity of transcriptional repressor octamer-binding transcription factor 1 (oct-1). J. Biol. Chem. 2013, 288, 14815-14823. [CrossRef] [PubMed]

37. Slominski, A.; Zmijewski, M.A.; Pawelek, J. L-tyrosine and 1-dihydroxyphenylalanine as hormone-like regulators of melanocyte functions. Pigm. Cell Melanoma Res. 2012, 25, 14-27. [CrossRef] [PubMed]

(C) 2019 by the authors. Licensee MDPI, Basel, Switzerland. This article is an open access article distributed under the terms and conditions of the Creative Commons Attribution (CC BY) license (http://creativecommons.org/licenses/by/4.0/). 